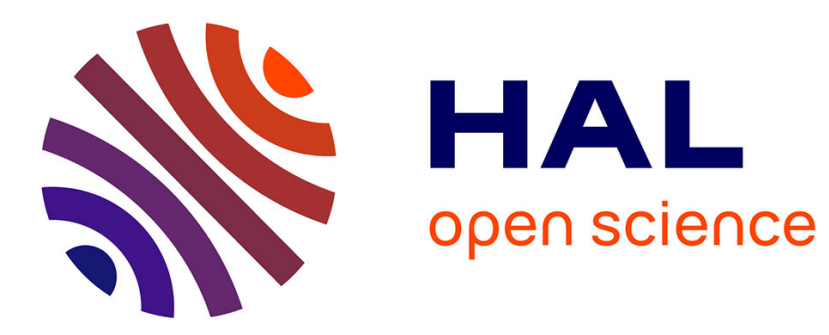

\title{
Casablanca racontée par ses vendeuses de lben : trois regards à la croisée de la ville et de la campagne
}

Yasmine Berriane, Leila Bouasria

\section{To cite this version:}

Yasmine Berriane, Leila Bouasria. Casablanca racontée par ses vendeuses de lben: trois regards à la croisée de la ville et de la campagne. Karthala. Casablanca: Figures et scènes métropolitaines, p.141-165, 2011, 2811104569. hal-03125615

\section{HAL Id: hal-03125615 https://hal.science/hal-03125615}

Submitted on 29 Jan 2021

HAL is a multi-disciplinary open access archive for the deposit and dissemination of scientific research documents, whether they are published or not. The documents may come from teaching and research institutions in France or abroad, or from public or private research centers.
L'archive ouverte pluridisciplinaire HAL, est destinée au dépôt et à la diffusion de documents scientifiques de niveau recherche, publiés ou non, émanant des établissements d'enseignement et de recherche français ou étrangers, des laboratoires publics ou privés. 


\section{Casablanca racontée par ses vendeuses de lben : trois regards à la croisée de la ville et de la campagne}

BERRIANE Yasmine \& BOUASRIA Leila

Il s'agit ici de la version preprint d'un chapitre dont la version finale et définitive a été publiée dans : M.Peraldi and M.Tozy (dir.), Casablanca : Figures et scènes métropolitaines, Paris : CJB/Karthala/CM2S, Collection Hommes et sociétés, 2011, p.141165.

\section{Les protagonistes}

Il est 7 heures 30 du matin, la ruelle commerçante faisant face au marabout ${ }^{1}$ de Sidi Messaoud se réveille au rythme des vendeurs et des premiers camions qui passent en soulevant la poussière à leur passage. En face des constructions maraboutiques, s'égrène une rangée de petits locaux principalement occupés par des vendeuses de $l b e n^{2}$. Nettoyant le sol, rinçant la vaisselle, préparant de nouvelles bouteilles à la vente, elles s'apprêtent à accueillir les premiers clients de la journée. Assises au bord du trottoir, d'autres femmes attendent, les bras chargés de bidons vides. Certaines discutent entre elles, et d'autres, le regard dans le vide, attendent, impassibles.

À l'approche de 8 heures, l'endroit s'anime lorsque deux vieilles fourgonnettes s'approchent. Leurs coffres sont remplis de grandes jarres en plastique ou en métal. C'est l'heure de la livraison de lait. Les femmes assises au bord du trottoir se lèvent et préparent leurs bidons en suivant du regard les livreurs qui, un carnet à la main, règlent leurs comptes avec les vendeuses dans les locaux. Rahma en fait partie et c'est depuis des années qu'elle se fait livrer par Haj Lkbir qui vient de la commune rurale d'Ouled $\mathrm{Hriz}^{3}$. D'un geste machinal, ce dernier lui remplit ses jarres vides et note sur son carnet la quantité livrée. Elle ne le paiera que dans deux jours lorsqu'elle aura battu le lait et vendu toute la marchandise.

\footnotetext{
${ }^{1} \mathrm{Au}$ Maghreb, un marabout est un saint local dont le tombeau est l'objet d'un culte populaire. On leur prête généralement des pouvoirs multiples; principalement ceux de guérir. Pour désigner un tel lieu de culte, on utilise le terme de 'seyyid' qui désigne le saint personnage dont le tombeau sert de base aux visites. Dans le vocabulaire courant le saint et le lieu se confondent.

${ }^{2}$ Le lben est une sorte de petit lait ou lait fermenté.

${ }^{3}$ Ouled Hriz est une commune rurale rattachée à la ville de Berrechid dans la région de la Chaouia-Ouardigha.
} 
Le conducteur de la deuxième voiture, Haj Brahim, se dirige vers l'autre bout de la rangée où se trouve le local de Mina qui, âgée d'une quarantaine d'années, est la plus jeune des vendeuses propriétaires de local. Le livreur fournissait déjà du lait fermenté à sa mère avant que celle-ci ne décède. Il se renseigne sur l'état de santé de Haj Selam, le père de Mina, avant de s'assurer que cette dernière compte bien, comme à son habitude, fermer son local le lendemain et se rendre en ville - à Bachkou ${ }^{4}$ - pour y vendre son lben. Elle acquiesce. Pour le lendemain, il lui faudra donc une livraison plus importante que d'habitude. Les affaires marchent mieux en ville.

Haj Brahim est l'un des principaux livreurs des vendeuses « sédentaires » de Sidi Messaoud qui sont servies en premier, compte tenu de leur ancienneté sur les lieux. En retournant vers sa voiture, il y trouve rassemblées trois autres vendeuses qui attendent les bras chargés de bidons. Celles-ci ne disposent pas d'un local. Elles iront vendre directement la marchandise en ville. Haj Brahim s'empresse de faire descendre ses jarres de lait fermenté pour aider les vendeuses à remplir leurs bidons et bouteilles vides. Ses gestes sont sans cesse interrompus par les lamentations des marchandes ambulantes voulant emprunter des jarres ou n'ayant pas suffisamment d'argent pour le payer. Les négociations fusent de toutes parts provoquant tantôt la colère du fournisseur qui a hâte de se frayer son chemin vers la ville, tantôt les moqueries et commentaires des vendeuses sédentaires siégeant souverainement dans leurs stands et observant la scène de loin.

Parmi ces vendeuses ambulantes, Habiba, loin d'attendre patiemment son tour, négocie à voix haute avec le fournisseur. Elle n'est pas satisfaite de la qualité du lben de la veille. Ses clients se sont plaints et elle ne peut pas se permettre de les perdre. D'un âge indéfinissable, le visage marqué par les années difficiles, elle est accompagnée de ses deux enfants en bas âge. Le plus grand s'amuse à arracher les plumes de deux poules emprisonnées non loin de là dans une caisse en bois. Le livreur ne semble pas enclin à discuter avec elle et préfère se concentrer sur la jarre qu'il est en train de lui remplir. Lorsqu'une charrette tirée par un mulet s'approche, Habiba demande au conducteur s'il va vers Sidi Maarouf. Ce dernier lance un regard irrité vers les deux enfants et la grande jarre mais cède face aux supplications de Habiba qui attirent l'attention des passants. Et la voilà partie à la rencontre de sa clientèle.

\section{Le lieu}

Cette scène matinale se déroule dans la principale ruelle commerçante du site maraboutique de Sidi Messaoud qui se trouve à l'extrême périphérie de Casablanca. Ce lieu de culte est situé sur la route de l'aéroport Mohammed $\mathrm{V}$ et dans une région qui porte le même nom que le saint qui y repose. A neuf kilomètres de la ville, le site qui domine d'un côté la ville de

\footnotetext{
${ }^{4}$ Bachkou est un quartier casablancais avec un bidonville du même nom qui se trouve encastré entre les deux quartiers résidentiels Polo et Oasis.
} 
Casablanca et de l'autre la plaine des Ouled Haddou ${ }^{5}$ est également un endroit où convergent les itinéraires des Casablancais empruntant le raccourci qui débouche sur Sidi Maarouf ${ }^{6}$ ou sur l'axe routier menant à Berrechid ${ }^{7}$.

L'origine du saint patron de ce lieu, Sidi Messaoud Ben Bouziane également surnommé Moulay Tadarte en allusion à l'endroit où serait enterré son père, reste floue ${ }^{8}$. Il n'existe que très peu de littérature sur ce lieu de culte ${ }^{9}$. Sidi Messaoud se serait très tôt distingué par ses dons de guérisseur qu'il utilisait pour soigner les personnes atteintes de folie. Quant au mausolée ${ }^{10}$, il daterait de la fin du XIXème siècle ${ }^{11}$ et continuerait à accueillir aujourd'hui des patients atteints de folie. Ces derniers sont alors enfermés dans les «chambres du pardon» (beyt laafou) accolées au sanctuaire en attendant leur guérison. La majorité des pèlerins et visiteurs vient pourtant simplement pour y déposer des bougies et s'y recueillir.

Cette zone périurbaine des Ouled Haddou est également ancrée dans la mémoire de tout Casablancais ayant eu l'habitude d'y passer les vacances de printemps. En effet, ce lieu de culte a également longtemps été célèbre pour les possibilités d'escapades qu'il offrait aux Casablancais en mal de verdure et de nature. Situé non loin de la forêt de Bouskoura, il était jadis exclusivement entouré de champs verts. La route n'était pas goudronnée et les vendeurs proposaient leur marchandise à l'ombre de leurs tentes... Peu de traces alors de la ville avoisinante.

Aujourd'hui, les vastes plaines de Sidi Messaoud disparaissent petit à petit derrière les nouvelles constructions dessinant les contours d'une urbanité encore «floue $»^{12}$. Bien que ses habitants n'ont de cesse de répéter que cette métropole géante approche de plus en plus vite et qu'elle va bientôt tout engloutir sur son passage, le ras de marée n'est percevable que par endroits. A cet endroit-charnière tantôt perçu comme un lieu de culte, tantôt comme un site commercial ou encore comme un lieu de sorties familiales du dimanche, l'urbain et le rural se côtoient et s'entremêlent encore à niveau presque égal.

\footnotetext{
${ }^{5}$ Ouled Haddou est l'ancienne dénomination (utilisée encore) attribuée aux plaines du Sud de Casablanca. Les habitants de Ouled Haddou - les haddawiyin - se disent être les vrais habitants (pure souche) de Casablanca.

${ }^{6}$ Sidi Maarouf est un quartier périphérique de Casablanca se situant sur la route de Bouskoura. Le nom est associé à Ouled Haddou (la commune de Sidi Maarouf-Ouled Haddou).

${ }^{7}$ Berrechid est une ville à une trentaine de kilomètres au sud de Casablanca relevant de la région ChaouiaOuardigh.

${ }^{8}$ Les uns le disent originaire de l'actuelle Algérie, d'autres le présument originaire du Tafilelt et d'autres enfin originaire de Taddart (situé à Ouled Haddou) où se trouve enterré son père.

${ }^{9}$ B. Etienne qui traite, en 1979, de plusieurs marabouts casablancais, nous livre les informations suivantes: « Mausolée de Sidi Messaoud avec annexes, tombeaux de Sidi Ali El Hajjun, Lalla Chaïba, Sidi M'Hamed Ben Amar; appartient à une famille casablancaise (Bou Amriyin) qui réclame ce Sidi M'Hamed comme leur grandpère, mais leur ancêtre (cherif) est enterré à Meknès (donc le corps n'est pas dans le Darih). Sidi Messaoud est en effet enterré dans le territoire de la tribu des Ouled Haddou sur la route de Mediouna. L'ancêtre de Sidi Messaoud, lui, est enterré à Seguiet el-Hamra, c'est pourquoi le nom exact du Saint est : S. Messaoud Moula Taddert » (Etienne, 1979, p.283).

${ }^{10}$ Tombeau du Saint-Patron qui est généralement sous forme de coupole.

${ }^{11}$ Bensaber, 2006.

${ }^{12}$ Benlakhlef; Zeghich ; Boukhemis, 2005, p.51.
} 
La petite coupole du sanctuaire en haut de la colline a été remplacée par une construction plus imposante au toit pointu tapissé de tuiles vertes et qui, du haut d'un long et large escalier, surplombe tout le site. Cette image imposante est troublée par la rangée de poteaux électriques qui se dressent en face du mausolée. Le long de l'escalier en béton, des palmiers encore jeunes ont été plantés et non loin de là, s'étend un tout petit cimetière, réservé aux descendants du Saint. Du haut du sanctuaire, le visiteur a une vue d'ensemble sur des champs avoisinants, des terrains vagues, des constructions en béton éparses et des pistes de terre fréquentées par des mobylettes, de vieilles voitures et des charrettes. Au loin, l'œil distingue clairement les constructions de la ville. En descendant le grand escalier en béton, on passe près d'une mosquée d'aspect récent et qui se dresse, imposante. Elle fut construite en 1994 par un bienfaiteur installé en ville mais originaire de la région. A droite, un parking est destiné aux visiteurs du site. Tout en bas de l'escalier, on se retrouve face à une route goudronnée qui est surtout utilisée par des camions se dirigeant vers la carrière de pierres située à quelques kilomètres de là, non loin de l'entrée de la ville et du quartier de Sbata.

En face du lieu de culte, de l'autre côté de la route, on distingue une rangée de petits magasins, des locaux et des gargotes construites en béton. Ces constructions forment un ensemble à deux faces, l'une donnant sur la rue et donc visible à la clientèle et l'autre cachée. La face visible est occupée par les locaux de nos vendeuses de lben qui y vaquent à leurs tâches habituelles de commerçantes. S'y active également un boucher, un épicier et quelques locaux fermés. Le tout est d'aspect plutôt soigné et propre. La face de l'ensemble tournant le dos à la rue est très différente. On y découvre plusieurs rangées de locaux en béton dans lesquels s'affairent, non loin d'une petite épicerie et d'une pharmacie, des ferronniers, des menuisiers, un fabricant de corbeilles en plastique recyclé et un coiffeur. De ce côté, le passant doit marcher sur de la terre battue. Par temps de pluie, de grosses flaques s'y forment et la boue se fait abondante. Par temps de sécheresse, une poussière de terre recouvre tout d'une fine couche rougeâtre qui se mélange à la suie des différentes machines. Ces dernières (marteaux, scies et chalumeaux) produisent un bruit insupportable.

C'est donc dans ce décor que Rahma, Mina et Habiba - nos trois protagonistes - se rencontrent, tous les matins, pour se ravitailler avant de partir, chacune de son côté et à sa manière, revendre sa marchandise à une clientèle majoritairement citadine. Dans tout Casablanca, le site de Sidi Messaoud est connu pour ses vendeuses de lben. Rahma la vendeuse « sédentarisée », Mina « l'ambulante à mi-temps » et Habiba «l'ambulante à plein temps » ont chacune une relation propre à la ville. Une relation qui dépend beaucoup de la manière qu'elles ont de pratiquer leur métier. A travers les souvenirs et la vie quotidienne de chacune de ces trois vendeuses se dégagent trois lectures différentes (tout en étant complémentaires) des mutations que connaissent les périphéries de la ville de Casablanca et des processus d'adaptation et de transformation de son milieu périurbain. 
Notre regard s'est porté sur deux grands axes tout au long de nos multiples rencontres avec les vendeuses. Nous nous sommes d'abord intéressées au lieu de départ: à Sidi Messaoud. Hormis le fait que ce soit l'endroit charnière où se rassemblent les acteurs principaux du circuit de commercialisation du lben, son histoire ainsi que les différentes étapes de son évolution nous aident à décrypter les nouveaux comportements des vendeuses sédentaires et ambulantes et les nouvelles dynamiques d'afflux de leur clientèle. Ensuite, ce sont les itinéraires des vendeuses ambulantes qui ont attiré notre attention : qu'est-ce qui motive les choix de leurs lieux de vente? De quelle manière s'approprient-elles l'espace et comment lui donnent-elles une fonction ? Enfin, au fil de leur ambulantage ${ }^{13}$, quelle relation entretiennentelles avec la ville?

\section{I- Rahma : la mémoire vivante du lieu}

Rahma doit approcher les soixante-dix ans, pourtant elle paraît en faire bien plus : le visage rond, boursouflé et ridé, le teint clair et le regard légèrement trouble, signe d'une cataracte naissante. Telles les femmes à la campagne, sa tenue est constituée de différentes couches superposées. Elle arbore généralement des collants ou encore un pantalon de pyjama, pardessus une robe d'intérieur à rayures recouverte d'un pull, le tout accompagné d'une large jupe ou d'un vieux caftan délavé protégé d'un grand tablier de cuisine attaché autour de la taille. Cette superposition d'habits doit avant tout être pratique, protéger du froid et ne pas gêner pendant le travail. Sa tête est recouverte d'un foulard. A la voir, on ne peut s'empêcher de se demander si elle ne s'habille pas de la même façon lorsque, de retour de son lieu de travail, elle se retrouve dans l'espace privé de sa maison.

Cette superposition du public et du privé se laisse également entrevoir dans la décoration et la disposition de son local. À première vue, l'intérieur de tous les locaux se ressemble: une table basse en bois au centre et une banquette plastifiée qui longe la paroi du fond. Bien que standard, cet ameublement confère à chaque local une petite touche d'intimité. C'est une salle à manger dans laquelle les «invités » se rassemblent autour d'une même table. Rahma a d'ailleurs décidé d'égayer cet intérieur par un assortiment de fleurs artificielles qu'elle nous montre avec fierté. Ce côté intime et privé des locaux est renforcé par l'accueil chaleureux et convivial des vendeuses. Rahma est attentive à toutes les allées et venues devant son stand. Elle refuse de s'asseoir pour nous parler. Elle doit rester vigilante, à l'affût de nouveaux clients. Quand elle en aperçoit un, c'est avec un signe de main convivial qu'elle tente de l'attirer vers son local «Mrahba bikum ${ }^{14}$. Installez vous. Reposez vous». Le tout donne à cette ruelle une atmosphère particulière d'intimité. Il semblerait presque que pour Rahma et

\footnotetext{
${ }^{13}$ Nous reprenons ici ce néologisme utilisé par J.Monnet pour faire la distinction entre le 'colportage' qui serait caractéristique de la commercialisation préindustrielle et le commerce ambulant qui se développe avec la métropolitsation contemporaine (Monnet, 2006).

${ }^{14}$ Signifie « soyez les bienvenus » en dialecte marocain.
} 
ses voisines, le local soit une extension de la maison, une sorte d'espace privé ou semi-privé où elles évoluent sans aucune gêne et que ceci justifierait et « excuserait », en quelque sorte, leur présence active en dehors du domicile.

Devant chaque stand, on aperçoit le même étalage de bouteilles en plastique vides attendant d'être remplies, alors que d'autres déjà pleines de lben sont prêtes pour la vente. Le reste du lait fermenté est stocké dans des jarres en plastique bleues qui se dressent non loin de deux grands plats en terre cuite qui sont remplis de semoule fumante (belboula). Au fond du stand de Rahma, trône un réfrigérateur, un privilège que n'ont pas toutes les autres vendeuses. En face, se trouve une mystérieuse petite pièce-grenier fermée par un rideau où elle semble garder ses provisions mais où il nous est absolument interdit d'entrer.

A chacune de nos visites, nous devons d'abord nous installer sur le banc du fond et consommer un bol de seykouk $k^{15}$. Rahma s'installe alors près de nous et se met à raconter. Elle aime répéter que les temps ont changé, que rien n'est plus comme avant. En mettant en rapport ce refrain avec l'histoire du lieu qu'elle nous décrit, nous nous rendons bien compte qu'il s'agit ici, outre la nostalgie toute naturelle d'une personne âgée, de l'expression d'un sentiment de frustration né d'une série de ruptures avec le passé. Des ruptures qui ont profondément transformé le site de Sidi Messaoud et la vie quotidienne de ses vendeuses de lben.

\section{Exode rurale et installation à Sidi Messaoud}

A l'époque du retour d'exil de Mohammed V en 1955, Rahma arrive avec son mari à Casablanca à la recherche d'un travail et s'installe avec 30 autres ménages (la plupart originaires des Rhamna ${ }^{16}$ et de Ouled Saïd ${ }^{17}$ ) dans un premier bidonville aux alentours de la ville et non loin d'un grand étang. Ils devront pourtant tous quitter cet emplacement un peu plus tard en raison des nombreuses inondations que connaît la région. Rahma ressasse ce déplacement avec une ironie empreinte d'émotion : que peut-on faire lorsque les sortilèges décident de votre destin? Quelque chose de magique expliquerait l'inondation qui a incité les habitants à se déplacer. Rahma se rappelle comment, à chaque fois qu'ils cimentaient le sol, l'eau rejaillissait de plus belle. Elle affirme avec conviction que lhaddawiyate se sont déversées sur eux (fadou alina). D'après Rahma, lhaddawiyate - issues des Ouled Haddou seraient originaires de ce site. Elle croit fermement au pouvoir posthume des rjal lblad (les gens du pays, les 'vrais' Casablancais) et perçoit l'inondation comme un signe qu'émet une terre pour rappeler aux nouveaux « locataires » la présence de ses enfants éternels.

\footnotetext{
${ }^{15}$ Il s'agit d'un mélange de semoule et de lait fermenté.

${ }^{16}$ Rhamna est une tribu installée au nord du Haouz de Marrakech, à Kelaat Sraghna.

${ }^{17}$ Ouled Said est le nom d'une autre tribu installée dans la province de Settat.
} 
Ainsi, 15 des 30 ménages seront déplacés à l'orée du site de Sidi Messaoud ${ }^{18}$. En accusant le makhzen ${ }^{19}$ d'avoir été négligeant à leur égard (ce makhzen qui semble toujours être perçu d'un œil méfiant), Rahma précise que tout ce que ce dernier a pu faire pour eux, c'était de mettre les baraques dans des camions et de les déplacer à un autre endroit. C'est ainsi que naîtra douar Mzabiyin ${ }^{20}$ où vivent aujourd'hui toutes les vendeuses de Sidi Messaoud.

En arrivant, Rahma découvre un site qui gravite autour du marabout qui n'était à l'époque qu'une coupole. Autour de la coupole se trouvaient surtout des champs. Quelques vendeurs ambulants se sont alors déjà établis face au site maraboutique. La spécialisation des commerçants évolue aussi avec le temps. Au tout début, avant que Rahma n'arrive, le lieu était réputé pour la vente de l'incontournable barouk ${ }^{21}$ qui accompagne chaque marabout : des amandes, des noix et des bougies. Vers les années 50, se seraient installés les vendeurs de légumes. A l'arrivée de Rahma sur le site, seules trois vendeuses de lben y circulaient déjà. Petit à petit, ce sera à leur tour d'avoir le monopole sur cet endroit. Rahma se joindra à elles.

Pour subvenir aux besoins de sa famille, elle décide en effet de suivre l'exemple des autres vendeuses déjà présentes sur le site. Rien de plus simple puisque la vente du petit-lait ne nécessite aucun capital et fait même appel à ses compétences domestiques de femme rurale. Comme toutes ces vendeuses qu'elle observait et qui semblaient bien gagner leur vie, elle savait traire une vache et se mettre sous une tente pour proposer sa marchandise.

Pendant les deux premières années de sa carrière de vendeuse de lben, Rahma avait l'habitude de s'asseoir sur une pierre (doukkana) et de mettre une couverture par terre sur laquelle elle étalait sa marchandise. Un trou creusé dans la terre permettait de garder le lben au frais et même si le soleil dérangeait les vendeuses et gâchait la marchandise, le pittoresque de l'installation plaisait aux clients venant de la ville pour changer d'air.

\section{Le site à son apogée}

La première rupture, qui coïncide avec la masira (la Marche Verte de 1975) ${ }^{22}$, est marquée par un intérêt accru pour la région et par l'apogée du site de Sidi Messaoud. De plus en plus

\footnotetext{
${ }^{18}$ La deuxième moitié des baraques sera déplacée vers Douar Ouled Bouabid Ouled Chheb, un bidonville périurbain connu aujourd'hui comme kariane Rhamna parce que la plupart des habitants y sont originaires de Rhamna.

${ }^{19}$ En Arabe signifie 'trésorerie' ou 'grenier' à l'origine du mot Français 'magazin'. Makhzen peut désigner plusieurs choses à la fois : par exemple la structure étatique qui se chargeait de la gestion financière ou plus généralement aujourd'hui le pouvoir central.

${ }^{20}$ Le douar est un petit village. Dans le cas des douars cités dans le texte suivant, il s'agit principalement de sortes de bidonvilles périurbains, situés en marge de la grande ville.

${ }^{21}$ Offrande faite au saint. Barouk dérive du mot arabe Baraka qui signifie, littéralement : « une bénédiction envoyée par Dieu ».

${ }^{22}$ Il s'agit d'une marche pacifique lancée en 1975 par le roi marocain Hassan II pour récupérer le territoire du Sahara occidental occupé par les espagnoles.
} 
de grands taxis le relient à la ville ${ }^{23}$ et de plus en plus de clients viennent à la recherche de la campagne. Un commerce parallèle se développe (de petites épiceries, la location de tentes pour les campings, des bouchers $)^{24}$ et les affaires marchent bien.

Afin de les aider à mieux organiser leurs métiers et afin de circonscrire leur forte concentration sur le site, la commune équipe les vendeurs de tentes. Selon la décision du président de la commune, ces dernières devaient toutes être bleues pour éviter les couleurs claires qui se salissent rapidement au contact de la boue et de la poussière. A chaque printemps, une grande foire se déroulait près de l'endroit où se trouve aujourd'hui la mosquée : un mois de festivités et une clientèle abondante ${ }^{25}$. Quand le camping se remplissait, les vendeurs de Sidi Messaoud sous-louaient des tentes pour gagner un revenu additionnel. L'été c'était au tour du moussem ${ }^{26}$.

\section{Le début de la crise}

Lancée à l'initiative du président de la commune, la construction des locaux en 1983 marque une nouvelle rupture dans l'évolution de Sidi Messaoud. Un tirage au sort est organisé pour distribuer ces locaux aux vendeurs. Les plus chanceux pourront s'installer dans ceux qui donnent sur la rue. Les autres devront se contenter des constructions beaucoup moins attractives donnant vers l'arrière. Rahma, elle, tire la bonne carte. Désormais munie d'une concession pour un local donnant sur l'avant, il lui faudra payer à la commune un loyer de soixante dix dirhams par mois. Tous les vendeurs ne tireront pourtant pas la bonne carte. Hamid le coiffeur, dont le local tourne le dos à la route tout en étant encastré entre deux ateliers de ferronnerie accuse les autorités d'avoir manipulé le tirage au sort et d'avoir favorisé de façon ciblée les femmes parce que ces dernières s'étaient montrées plus coopératives pendant les élections communales. D’après lui, ces mêmes décideurs se seraient abattus sur le site comme le mauvais sort et en auraient « renversé la face » ( « qalbou loujeh dyal Sidi Messaoud»).

A écouter la suite de l'histoire que nous relate Rahma, on en arrive en effet à croire à l'existence d'un tel mauvais sort. Après le meurtre d'un jeune garçon non loin du marabout, la

\footnotetext{
${ }^{23}$ B.Etienne recensant les taxis desservant Sidi Messaoud en 1979, écrit: « 12 à 18 taxis hippomobiles et 6 taxis automobiles se tiennent en permanence le samedi/dimanche, et vont et viennent sans cesse », (Etienne, 1979, p.283).

${ }^{24}$ B.Etienne recensait en 1979 une centaine de commerçants à Sidi Messaoud : « deux aèdes, trois restaurants, trois marchands de brochettes ou de beignets, un boucher, trente marchandes de lait, petit lait et saïqûk, deux épiciers, deux marchands de légumes, deux marchands de volailles, deux de charbon de bois et Kanûn, etc..., sans compter les chauffeurs de taxis (automobiles et voitures à chevaux). » (Etienne, 1981, p.273-274).

${ }_{25}$ «Il y a même un mini parc d'attractions avec balançoires! Les gens viennent camper littéralement. D'autres y passent en rentrant du bled [fortement concurrencé par la mer en été], il n'est pas rare de décompter plusieurs milliers de personnes. » (Etienne, 1979, p.283).

${ }^{26} \mathrm{C}$ 'est une manifestation culturelle et religieuse rendant hommage à un saint homme en célébrant des processions vers son sanctuaire. Ces rassemblements dépendent des conditions locales (récoltes) ainsi que du calendrier lunaire.
} 
région est catégorisée comme dangereuse. Les campings ne sont désormais ouverts que pendant la journée. La nuit, il faut rentrer chez soi. Les autorités policières deviennent plus présentes sur le site. Une époque marquée par l'insécurité et le manque de confiance commence. Vient s'y ajouter la sécheresse des années 1980 : la campagne change de visage et devient moins attrayante. Enfin, la construction de locaux n'a pas que des effets positifs : ils offrent des commodités pour les vendeurs, certes, mais ils ne conviennent plus à la clientèle citadine qui recherche un espace rural où prédomineraient la nature et la simplicité. Ils sont alors nombreux à reprocher à Rahma de ne plus vendre ses produits sous une tente.

Enfin, les locaux et la construction de la grande mosquée au début des années 1990 ont pris une grande partie de la place disponible pour l'organisation de la foire printanière qui finit par disparaître. La clientèle diminue de plus belle d'autant plus que le lieu a perdu tout son charme mystique. Pour couronner le tout, depuis quelques années, une nouvelle composante s'est imposée sur le site : chassés de la ville à cause du bruit qu'ils provoquent, et attirés par les locaux vides et bon marché, plusieurs ferronniers sont venus trouver refuge en face du marabout de Sidi Messaoud. Aujourd'hui, Rahma estime que 90\% de la clientèle du lieu vient pour les ferronniers. Mais depuis que ces derniers ont commencé à s'installer, il y a une dizaine d'années, le site a définitivement perdu son charme rural explique t-elle. Elle fronce les sourcils lorsque le bruit provenant d'une scie électrique devient presque intenable et ajoute avec agacement: « Nous n'avons aucun contact avec eux ».

\section{2- Une nouvelle ancienne pratique du métier : l'ambulantage ${ }^{27}$}

\section{Le commerce ambulant : une pratique ancienne}

Ces mutations que subit le lieu ont redonné vie à la pratique ambulante du métier de vendeuse de lben. Rahma, encore jeune, avait en effet l'habitude d'aller rejoindre une autre clientèle dans les régions rurales avoisinantes. L'organisation des moussems lui offrait l'occasion de rompre avec la routine de vente quotidienne en proposant ses produits sous une tente à partir de laquelle elle pouvait observer les fantasias $^{28}$ et les folklores traditionnels. Selon elle, depuis que ces événements se font de moins en moins réguliers en raison de la sécheresse, la tradition tend à se perdre. Les déplacements en ville étaient également courants. Les vendeurs ambulants accédaient jusqu'à Bab Marrakech ${ }^{29}$, au cœur même de Casablanca. Aujourd'hui un tel déplacement serait impensable. Des charrettes à mulet en plein centre de la ville ? Impossible.

Au fil des années, Rahma a acquis à Sidi Messaoud une clientèle fidèle qui lui permet de subvenir à ses besoins d'une façon régulière. Enfin, deux de ses enfants vivent aujourd'hui à

\footnotetext{
${ }^{27}$ Pour plus de détail sur l'emploi de ce néologisme, voir note p.5.

${ }^{28}$ Une fantasia est une démonstration de cavaliers.

${ }^{29}$ Bab Marrakech est l'ancienne médina de Casablanca.
} 
l'étranger et lui envoient régulièrement de l'argent. Elle dispose de tout ce dont elle a besoin et ne doit plus quitter son local pour vendre ailleurs.

D'ailleurs, qu'irait-elle faire en ville? Tout autour des petits commerces de Sidi Messaoud, un réseau de services informels s'est mis en place. Les jeunes des environs gagnent leur pain en ravitaillant les vendeuses en eau fraîche et en bouteilles vides. Les locaux étant équipés d'électricité mais pas d'eau courante, les commerçants gravitant autour du site doivent recourir à la petite source (laaouiyna) qui se trouve non loin de là. La livraison d'un baril de 200 litres d'eau coûte dix dirhams et peut tenir plusieurs jours. Quant aux bouteilles en plastique vides, les jeunes les collectent un peu partout, dans les environs et en ville, et viennent les revendre aux commerçants à vingt centimes la bouteille.

Les rares déplacements de Rahma se limitent aujourd'hui aux souks ruraux avoisinants ${ }^{30}$ (dans lesquels elle s'approvisionne en œufs frais et en semoule), aux visites à sa fille mariée à Aïn Chock $^{31}$, aux médecins et aux hammams de Sidi Maarouf. Toutes ses sorties ne la font guère rentrer plus profondément en ville.

\section{Mina : entre Sidi Messaoud et Bachkou}

Le teint basané rappelant ses origines de la région d'Essaouira au Sud du Maroc, Mina a aujourd'hui quarante cinq ans et a grandi entre les baraques de Douar Mzabiyin et les bidons de lait fermenté du local de sa mère. Bien que beaucoup plus jeune que Rahma, Mina ne peut s'empêcher de nous parler encore et encore de ce « bon vieux temps » qui a marqué l'apogée du site. Selon elle, Sidi Messaoud a énormément changé, et ce serait surtout, explique t-elle, la faute aux «ikhwaniyin » (les islamistes). Ils auraient tellement prêché contre les marabouts que, maintenant, les gens n'y croient plus. Et puis c'est aussi la faute de cette nouvelle génération ( had ljil jdid) qui a perdu la foi (niya). En disparaissant, la foi a également emporté une bonne partie de la clientèle!

Mina sait qu'elle pourrait vendre sa concession à un prix intéressant. L'intérêt des forgerons et menuisiers est grand pour le site de Sidi Messaoud qui se trouve suffisamment éloigné de la ville pour ne pas déranger ses habitants et suffisamment proche pour attirer la clientèle. On a proposé entre 12000 et 13000 dirhams à sa voisine Fatima qui attend de voir les prix monter à 24000 dirhams avant de vendre et de s'installer définitivement à Sidi Maarouf. Mais bien que désenchantée par les affaires qui vont mal, Mina refuse l'idée de vendre son local pour le moment. Consciente de son emplacement stratégique, elle préfère patienter. En attendant, elle a une solution de rechange qui lui permet de compenser le manque de clients : tous les vendredis, elle enfile une vieille djellaba par-dessus sa tenue d'intérieur, remplit toutes ses

\footnotetext{
${ }^{30}$ Elle dit préférer fréquenter les souks ruraux comme celui de Sidi Mesaoud (les dimanche), de Bouskoura (le mardi), de Mediouna (le jeudi), et de Berrechid (le lundi).

${ }^{31}$ Ain Chock se situe au sud-est de Casablanca.
} 
jarres de lben, achète chez le fournisseur trois kilos de beurre beldi, descend la grille en fer qui ferme l'entrée de son local et se hisse sur la charrette conduite par son père. Puisque les habitants de la ville ne viennent plus chercher leur lben à Sidi Messaoud, père et fille vont à leur rencontre en ville.

Depuis des années, ils sont fidèles au même emplacement en ville : devant la petite mosquée du bidonville de Bachkou, non loin de la gare d'Oasis. Cet emplacement idéal attire une large clientèle qui, après la prière du douhr, s'empresse d'acheter une bouteille de lben pour accompagner le couscous du déjeuner. La vente à Bachkou est particulièrement intéressante: Mina arrive à vendre en quelques heures jusqu'à quarante litres à cinq dirhams le litre alors qu'à Sidi Messaoud, en une journée, elle ne vend que dix litres à quatre dirhams le litre.

Sur les traces de Mina, nous avons beaucoup de mal à repérer l'entrée du bidonville qui se trouve être encastré entre les quartiers les plus chics de Casablanca (Oasis, California et Polo). La concentration de grandes villas de haut standing à l'endroit indiqué par Mina nous désoriente. Ce n'est qu'après avoir tourné en rond pendant une demi-heure, qu'un jeune passant nous indique l'entrée principale du bidonville: tourner à gauche au niveau de la téléboutique. Nous y découvrons une piste de quelques mètres qu'il faut longer avant de se retrouver nez à nez avec une petite artère commerçante très animée qui traverse un ensemble de baraques construites en tôle ou, plus rarement, en brique ${ }^{32}$. Le tout est entouré d'une clôture en dur, sorte de cache-misère sensé séparer le bidonville des villas qui se trouvent en face. Cette enclave au milieu de la ville qui fait se côtoyer misère et opulence est surprenante.

Tout le long de cette ruelle, nous retrouvons de petites épiceries et toutes sortes de vendeurs ambulants. Lorsque nous nous renseignons chez le premier épicier pour savoir si des femmes viennent vendre ici du lben, une petite fille s'écrie : «Allez chez Haj Selam, je te jure qu'il n'ajoute pas d'eau à son lait! ». Nous reconnaissons le nom du père de Mina et la petite fille s'empresse de nous mener jusqu'à cette dernière. Toujours habillée de sa djellaba, nous la trouvons assise sur un tabouret en face d'une toute petite mosquée. Elle a disposé en face d'elle la grande jarre de lben et plusieurs bouteilles préalablement remplies. Elle est entourée de nombreux clients qu'elle semble bien connaître. Certains sont munis d'une cruche ou d'un récipient métallique dans lequel ils se font verser le lben directement. En face d'elle, de l'autre côté de la petite rue, se trouve son père, allongé sur la charrette en bois. Il fait une petite sieste. À 14 heures, elle a tout vendu. Père et fille peuvent reprendre la route vers Sidi Messaoud.

\footnotetext{
${ }^{32}$ Les habitants de ce bidonville attendent d'être relogés mais c'est l'un des bidonvilles qui connaît les négociations les plus ardues quant au plan de recasement, vu l'importance stratégique de son emplacement à l'intérieur de la ville.
} 


\section{Habiba : l'ambulantage à temps plein}

De loin, n'apparaissent que des pieds chétifs dépassant d'une installation pour le moins expérimentale: un parasol bariolé auquel est attaché un bout de tissu à rayure amarré au sol par une branche d'arbre en guise de mur latéral. Le reste de l'étalage est plus typique : une table basse surmontée de bouteilles remplies de lben, dans un coin, un grand sachet orange plein de bouteilles vides, une bassine pour laver les ustensiles et la partie supérieure d'un couscoussier recouvert d'un tissu. En entendant nos pas, Habiba, assise par terre, tend la tête vers l'extérieur dévoilant son visage maigre. De sa voix aigue, elle laisse échapper un cri de joie. C'est la deuxième fois que nous venons lui rendre visite. La peau brune, d'une maigreur frappante, sa dentition supérieure a presque entièrement disparu. Portant une robe de chambre rouge serrée d'une corde fine à la taille, elle arbore sur la tête plusieurs foulards superposés et dans les bras son dernier-né, âgé de huit mois.

Habiba est marchande ambulante à plein temps. Originaire de Ouarzazate, elle est arrivée à Casablanca à l'âge de treize ans pour s'occuper des enfants de sa sœur installée à douar Mzabiyin. Mariée quelques années plus tard, elle s'installe dans le même douar avec son mari. Travaillant, au début, comme femme de ménage dans le quartier huppé de California, elle doit arrêter cette activité après la naissance de son premier fils, âgé aujourd'hui de quatorze ans. Une voisine lui conseille alors de suivre l'exemple des habitantes du douar et de tenter sa chance comme vendeuse de lben. Habiba se rend à Sidi Messaoud et observe les marchandes. Elle commence par acheter, à l'insu de son mari, de toutes petites quantités qu'elle vend aux jeunes étudiants qui viennent s'isoler près du site maraboutique. Petit à petit, Habiba s'éloigne de Sidi Messaoud à la recherche de sa clientèle. Lorsque ses entrées d'argent deviennent plus substantielles, son mari accepte de la laisser acheter une vieille charrette à bras et à deux roues et l'accompagne de temps en temps pour l'aider à tirer ce dernier. Avec l'aide de son époux, elle s'aventure de plus en plus souvent en ville.

Aujourd'hui, sa semaine est structurée en fonction des fluctuations de sa clientèle et de ses possibilités de transport. Le dimanche c'est journée de souk à Sidi Messaoud, Habiba s'installe alors près de la route principale qui longe la mosquée d'Lhamd. Le vendredi et le samedi, c'est au tour du petit souk de Sidi Maarouf et le restant de la semaine, elle s'installe près de la gare de Nassim $^{33}$ entre le quartier résidentiel et la zone industrielle. Portant son bébé sur le dos et chargée par son dispositif de vente, ses déplacements dépendent de l'humeur et du temps libre de son mari qui l'aide à tirer la charrette à bras. Pour l'instant, elle ne peut que rêver de pouvoir s'acheter un âne ou un mulet lui permettant de se déplacer sans restrictions et peut être de s'aventurer encore un peu plus en ville. Ensuite, s'enthousiasme- t-

\footnotetext{
${ }^{33}$ Nassim ville nouvelle est rattaché au territoire communal de Lissasfa et Dar Barazza. Elle se situe à proximité des berges de l'Oued Bouskoura, sur la route d'El Jadida. (Azzar, 2001, p.80).
} 
elle, elle se procurera une tente. Une belle grande tente qu'elle utilisera pour faire le tour des souks avoisinants et pour vendre également des tagines, du thé et des sardines grillées...

Le jour de notre deuxième rencontre, à l'ombre de sa tente de fortune, Habiba est encore loin de son rêve. S'affairant autour de son petit stand mobile, elle garde un œil sur son fils de 5 ans qui joue avec les enfants des autres vendeurs. Habiba dont la silhouette fragile ne manque pas de vitalité, conjugue ses différents rôles avec une grande délicatesse. En vendeuse éloquente, et dans un sursaut d'énergie, elle réussit à servir plusieurs clients à la fois en magnétisant par ses paroles ceux qui attendent. Sa voix puissante se fait entendre malgré les pleurs de son bébé et les piaillements des autres enfants. D'un geste ébauché affectueusement, elle marque souvent un arrêt pour allaiter son petit qui continue à pleurer malgré les efforts de Habiba de lui presser le lait de son sein. Ses gestes maternels contrastent avec l'air dur qu'elle arbore en s'adressant à certains de ses clients qui lui déplaisent : «ceux qui te disent qu'ils viendront payer plus tard et ne reviennent plus! » ou encore «ceux qui ne viennent que pour poser des questions idiotes sans rien acheter et qui cachent l'étalage aux yeux des passants!» Enveloppée de vêtements, comme pour effacer toute trace de féminité qui risque de compromettre le rôle qu'elle joue, Habiba s'adresse aux clients sur un ton ferme frôlant parfois l'agressivité.

\section{3 - La vente du produit : approvisionnement et « gages de confiance »}

Nos trois vendeuses proposent un assortiment de produits très semblables : du lben, de la semoule pour la mélanger au lait fermenté et faire du seykouk, du beurre beldi ${ }^{34}$ et des œufs durs. Évidemment, le local de Rahma et de Mina leur permet de vendre de plus grandes quantités et éventuellement de diversifier leur offre. C'est le cas de Aïcha par exemple, voisine et amie de longue date de Rahma. Profitant d'avoir un très grand local, elle propose également des mkhamer $^{35}$ pour le petit-déjeuner, des tagines pour le déjeuner, du thé, une cabine téléphonique et un téléviseur. Avec l'aide de ses fils, elle a agrandi son magasin initial en y incorporant trois autres locaux. Rahma secoue la tete en signe de désapprobation: la pauvre Aïcha ne fait que courir. Elle est seule à devoir discuter le prix du lait livré, fabriquer le lben, cuisiner et garder un œil attentif sur les allées et venues au sein de son local. Un seul fils l'aide à servir les clients. C'est beaucoup trop difficile à gérer. Rahma préfère miser sur une palette simple de produits et une clientèle limitée mais fidèle.

Pourtant, cette fidélité n'est pas garantie. Elle bute principalement face à la mauvaise réputation qui entoure les produits proposés par les marchandes. La méfiance des clients se porte autant sur l'origine de ces produits que sur leur qualité. Toutes sortes d'histoires à en perdre l'appétit circulent sur la production du lben et sur les différentes méthodes de rallonger

\footnotetext{
${ }^{34} \mathrm{Du}$ beurre fabriqué à la manière traditionnelle.

${ }^{35}$ Une sorte de galette de pain fine.
} 
le lait en y ajoutant de l'eau (pas toujours propre) et de la poudre de lait. Le résultat en serait un produit frelaté, sale, et en aucun cas « authentiquement rural ».

Hamid, le coiffeur de Sidi Messaoud qui a grandi et vécu toute sa vie dans la région, s'empresse de nous présenter sa propre version. Les fournisseurs sont en fait des citadins qui achètent les restes de vieux lait chez des sociétés de production de produits laitiers. Chez eux, en ville, ils en font du lben. Ensuite, ils l'acheminent jusqu'à Sidi Messaoud où les femmes des environs l'achètent pour le revendre aux citadins. Les unes le revendent sur place dans leur local et les autres se déplacent jusqu'en ville à la recherche de la clientèle. C'est un cercle parfait précise-t-il avec cynisme : le lait vient de la ville puis passe par la campagne pour pouvoir être revendu en ville.

Face à cette mauvaise réputation qui entoure le produit qu'elles commercialisent, nos trois vendeuses ont élaboré différentes stratégies de vente jouant sur plusieurs registres à la fois.

\section{Le triple gage de confiance de Rahma : ruralité, propreté et lmakina}

A soixante dix ans, Hajj Driss fait partie, comme Rahma, des premiers habitants du bidonville rural appelé Douar Mzabiyin. Chaque matin, il se rend chez elle pour lui livrer les quelques litres de lait produits par ses deux vaches. Bien que sa production ne couvre qu'une toute petite partie de l'offre de Rahma, cette dernière insiste pour nous présenter Hajj Driss comme son livreur, vieil ami et voisin digne de confiance. Ses deux vaches sont présentées comme la preuve irréfutable de l'origine rurale et traditionnelle des produits de Rahma. Quant aux deux autres livreurs, elle ne les mentionne jamais par leurs noms mais toujours par leur région (rurale) d'appartenance. Ainsi, la plus grande partie de son lait lui vient d'un fournisseur de Oulad Hriz ${ }^{36}$. Quant aux autres livreurs, ils viennent respectivement de Ouled rrar $^{37}$ et de Bouskoura $^{38}$.

L'origine rurale de sa matière première ainsi authentifiée, Rahma n'a de cesse de mettre en avant les avantages du monde rural dans lequel elle situe incontestablement Sidi Messaoud. Elle y oppose lemdina (la ville) qui se trouve délimitée par les secteurs périphériques qu'elle fréquente le plus comme Sidi Maarouf, Aïn Sbaa ou encore Aïn Chock. Elle insiste sur le contraste entre cette ville bruyante et polluée et le calme de la campagne où elle vit et travaille. Là au moins, les valeurs traditionnelles subsistent, l'air est respirable, la nature et le calme sont garantis. Nous ne pouvons alors nous empêcher de sourire lorsque, en même temps qu'elle loue les avantages de la vie de campagne, un camion passe devant le local en soulevant un nuage de poussière et, qu'à quelques mètres de là, le bruit strident d'une scie se fait à nouveau entendre. Imperturbable, élevant légèrement la voix pour se faire entendre,

\footnotetext{
${ }^{36}$ Ouled Hriz est une commune de Berrechid rattachée à la région de la Chaouia-Ouardigha.

${ }^{37}$ Ouled Jrar est une commune située au sud de Casablanca, à Had Soualem.

${ }^{38}$ Bouskoura se situe au sud de Casablanca vers la route de Berrechid.
} 
Rahma continue son argumentation: les produits provenant de la campagne sont bien meilleurs que ceux de la ville. De toute façon, les citadines ne savent plus cuisiner correctement. La plupart ne savent même plus préparer la semoule pour le couscous. Le gaz ne peut en aucun cas donner le même goût au plat que le tagine cuit sur du feu de bois. C'est bien normal alors, que ses clients se déplacent jusqu'à Sidi Messaoud à la recherche d'un goût et d'une authenticité qu'ils ne retrouvent plus dans leur entourage citadin et qu'elle, Rahma, peut leur offrir.

Mais l'origine rurale et "authentique» de ses produits est loin d'être suffisante pour convaincre le client citadin. Ainsi, à l'instar des autres vendeuses, Rahma donne toujours l'air d'être très occupée. Quand elle ne sert pas un client, elle nettoie soigneusement des bols et des cuillères ou balaie les débris du sol à l'aide de gros bouquets de thym sauvage. À intervalles réguliers, c'est avec beaucoup d'eau qu'elle nettoie le sol de son local. Ce nettoyage inlassable et démonstratif parait surprenant au premier abord, mais fait partie d'une stratégie de vente qu'elle décrit de la façon suivante : «La propreté, c'est ce que cherchent les gens. Si ce n'est pas propre, ils ne vont pas s'arrêter ».

Quant à son dernier, et plus important, gage de confiance c'est la fameuse makina ${ }^{39}$. Autrefois, elle utilisait la chekwa en cuir (l'outre) qu'elle secouait manuellement pendant des heures pour baratter son lait. Aujourd'hui, la production de lben est beaucoup plus simple. Depuis l'arrivée de l'électricité à Sidi Messaoud et l'installation de compteurs dans les locaux, la chekwa a laissé place à la machine qui facilite la tâche et allège une grosse partie de ses corvées quotidiennes.

Pour fabriquer du lben, il faut conserver le lait à une température moyenne, dans des jarres spéciales réservées à cet usage. Le temps du repos dépend des saisons; en été le lait peut être caillé en une journée mais cela peut prendre deux à trois jours en hiver. Le lait est caillé ( $r a b)$ quand une couche de crème jaunâtre remonte à la surface. Il s'agit alors de verser la partie liquide dans le tonneau adapté au moteur de la machine qui va battre le lait. Après quinze minutes de barattage, Rahma ouvre la jarre pour vérifier si tout le beurre est monté à la surface. Même si son métier paraît facile, il obéit à une série de petites astuces garantissant la réussite du produit : «l'hiver, ajoute toujours un peu d'eau chaude au lait, l'été il faut ajouter de l'eau froide. C'est primordial. » Le barattage continu jusqu'à ce que la crème se transforme en beurre. Ce dernier est alors retiré pour le laisser égoutter dans le réfrigérateur. Rincé à l'eau salée, il sera ensuite empaqueté dans un sachet plastifié. Le lben, quant à lui, reste dans le tonneau et sera vendu à la bouteille ou au verre.

Malgré les frais d'électricité très élevés qui résultent de cette forme de production, Rahma a fait le choix de continuer à utiliser lmakina. Son calcul est encore une fois bien réfléchi : puisque les clients cherchent un lben de qualité, ils reviendront chez elle si elle leur offre un

\footnotetext{
${ }^{39}$ Ce mot signifie « la machine » en dialecte marocain.
} 
bon produit. Elle préfère miser sur la satisfaction des clients au lieu de se reposer sur « l'argent facile ». La plupart de ses collègues - «les fainéantes » - ont fait le calcul inverse. L'utilisation de la machine coûte cher et prend du temps. Elles préfèrent acheter le produit tout prêt auprès des livreurs.

Ainsi, la machine est devenue une sorte de label, une façon de distinguer entre les vendeuses sérieuses, les «transformatrices», qui produisent elles-mêmes leur lben (donc celles qui détiennent une machine) et les « revendeuses » (sans machine) qui ne peuvent pas fournir de garanties suffisantes quant à l'origine de leur produit. Il en résulte un jeu amusant de cachecache et de formulations vagues visant à duper le client venant de la ville à la recherche de produits sains et authentiques. Ainsi, après notre première visite à Sidi Messaoud, nous étions persuadées que toutes les vendeuses disposaient d'une machine mais que presque toutes ces machines se trouvaient, par un pur hasard, ou bien dans le domicile des vendeuses ou encore étaient en réparation. Ce n'est qu'au fil des rencontres que nous avons compris que seules Rahma et sa voisine en disposaient d'une.

\section{Un gage de confiance alternatif: le poids de l'héritage}

Nous n'avons jamais eu l'occasion de voir lmakina de nos deux autres marchandes. Mina nous a pourtant raconté à chacune de nos visites une histoire différente justifiant l'absence de cette dernière : laissée à la maison, en réparation, prêtée à une voisine. Ce n'est que le dernier jour que, ne sachant probablement plus vraiment à quelle version nous nous étions arrêtée la dernière fois, elle raconte ne pas avoir de machine et acheter le lait fermenté tout prêt chez le livreur. Elle se rattrape pourtant en insistant sur le fait que non seulement ce dernier s'approvisionne chez les paysans de Ouled Jrar mais, qu'en plus, c'est également un cousin lointain. Quelle preuve supplémentaire faudrait-il pour garantir un produit sur et de qualité! Habiba ne bénéficie pas non plus d'une machine mais elle n'essaie pas de le cacher. Se faisant appeler lhaddaouia par allusion aux habitants originaires de la région de Casablanca, elle insiste également à tout bout de champ sur l'origine authentique de son produit. La preuve en est évidente : non seulement elle serait, grâce à ses origines, une des dernières vendeuses authentique de la région, mais, en plus, elle ne vend que de toutes petites quantités - la garantie pour une production non industrielle!

Mina à l'avantage de pouvoir s'appuyer sur le réseau de clientèle préalablement constitué par ses parents. Après avoir hérité du local de sa mère à Sidi Messaoud, elle a également hérité du vieil emplacement de son père qui, avant de vieillir et de tomber malade, se chargeait luimême de la vente ambulante dans le bidonville de Bachkou. Au fil des années, son père s'est construit un solide réseau de clientèle qu'il a transmis à sa fille. Bien que Mina ait pris la relève, son père continue pourtant à l'accompagner chaque vendredi. De ce fait, les habitants de Bachkou l'appellent « la vendeuse de lben qui vient avec son père ». Ce dernier se charge 
de conduire la charrette tirée par un mulet et reste assis ou allongé, gardant un œil vigilant sur sa fille pendant que cette dernière se charge de la vente. Elle nous raconte que la présence du père est nécessaire parce que les gens de Bachkou « ne sont pas faciles » (nass bachkou saâb). Par contre, la vente fonctionne beaucoup mieux depuis que Mina a pris la relève. Puisque ce sont surtout des femmes qui viennent faire les courses et acheter ce genre de produit, il est préférable qu'une femme les serve. Elles se sentent plus à l'aise.

Comme Habiba ne peut pas miser sur un capital social comparable à celui de Mina, elle s'est créé une lignée héréditaire sensée donner plus de poids à son activité de marchande et à la qualité de son produit. Les versions que nous avons récoltées auprès d'elle se contredisent toutes mais elles ont toutes un point commun: la création d'une chaîne de succession légitimant sa présence à son emplacement de Nassim et inscrivant son activité dans la durée. Ainsi, lors de notre première rencontre, elle nous raconte comment elle avait hérité l'endroit de sa tante, cette dernière ayant succédé elle-même à un autre membre de la famille. Habiba raconte fièrement qu'elle est la troisième de sa famille à vendre le lben à cet endroit. Quelques semaines plus tard, elle nous raconte qu'elle a pris la relève de sa voisine et amie qui avait pendant de nombreuses années vendues son lben près de la gare de Nassim. Plus tard nous apprenons qu'elle a été obligée de se rabattre sur cet emplacement parce qu'elle avait du mal à diversifier sa clientèle... La recherche de ses points de vente actuels n'a effectivement pas été très facile.

\section{4- Choix et appropriation de l'espace}

\section{Une appropriation difficile de l'espace}

C'est en riant que Habiba nous relate comment elle s'est aventurée pour la première fois en dehors de Sidi Messaoud. Elle s'était installée le long de la première route principale et avait passé la journée debout, sa marchandise entre les mains, faisant signe aux passants de s'arrêter. Ce jour là, elle n'avait rien vendu.

Le deuxième jour, et sur les conseils du livreur de lait, Habiba s'est rendue près de la mosquée de Sidi Maarouf. Cet emplacement est stratégique, car les habitants du quartier venant à la prière en profitent toujours pour se désaltérer d'un verre de lben ou d'un bol de seykouk. La place juste en face de la sortie de la mosquée était déjà occupée par une vendeuse qui voit d'un mauvais œil l'arrivée de Habiba. Elle lui fait comprendre rapidement qu'elle ne peut pas installer son étalage juste à côté d'elle et qu'elle doit s'éloigner de quelques mètres, vers le bas de la route. Bien qu'elle se soit exécutée, les rivalités entre les deux vendeuses créent des conflits que Habiba, qui n'a pas encore un réseau de clientèle stable, ne se sent pas en mesure d'affronter. Elle se voit alors contrainte de s'éloigner de la mosquée pour tenter sa chance sur 
la route principale qui longe la mosquée el-Hamd, non loin du site de Sidi Messaoud et à l'orée du quartier California.

Cette route à deux voies arbore un aspect différent les dimanches quand les vendeuses s'y alignent pour proposer leur marchandise. C'est alors la journée du souk à Sidi Messaoud et les habitants des alentours, principalement des quartiers chics de l'Oasis et California, s'y approvisionnent. Pour prendre la petite piste qui mène vers le parking du souk, les voitures doivent longer la route au bord de laquelle sont installés les étalages des marchandes de lben. Cet emplacement présente l'avantage d'offrir une clientèle diversifiée. Mais Habiba ne pourra finalement s'y installer que les dimanches parce que : "C'est une route importante par laquelle passent les hauts responsables alors ils n'aiment pas qu'on y reste. Pendant la semaine, nous devons nous trouver d'autres endroits. Il n'y a que le dimanche que nous sommes autorisées à nous installer ici. ». Bien que certaines vendeuses soient prêtes à prendre le risque d'y rester pendant la semaine, Habiba a trop peur des conséquences. Dernièrement, une des vendeuses s'est vue confisquer tout son matériel par des agents d'autorité.

En semaine, quelques vendeuses trouvent refuge un peu plus loin, sur la route de Bouskoura. Cet endroit est surnommé laghouiba (la petite forêt) alors que seuls quelques arbres chétifs s'y dressent. Habiba préfère ne pas s'aventurer toute seule à cet endroit parce que sa voisine s'y est fait agresser. Elle retente donc sa chance à Sidi Maarouf. Cette fois, au petit souk qui s'y tient tous les jours. Cet endroit reste facile d'accès: lorsque son mari ne peut pas l'accompagner pour tirer la charrette, elle peut également se faire conduire, pour quatre dirhams, par une des innombrables charrettes à mulet qui font le trajet entre Sidi Messaoud et Sidi Maarouf.

En arrivant, elle est la seule vendeuse. Elle pressent enfin une possibilité de se construire un réseau de clientèle bien à elle. Pourtant, ce n'est qu'avec beaucoup de difficultés qu'elle y arrivera. Après seulement quelques jours, les clients, mécontents, viendront lui expliquer que son lben n'est pas de bonne qualité et qu'il est dilué. La clientèle du souk de Sidi Maarouf n'est pas facile. Les habitants de cette partie de la ville sont majoritairement originaires de la campagne. Ils apprécient le lben mais ils sont bien plus exigeants que la clientèle purement citadine. Suivant leurs conseils, elle change de livreur. Aujourd'hui, ses clients sont satisfaits du produit. A l'observer, on sent qu'elle s'est faite sa place au sein du petit souk. De temps en temps, mais c'est très rare, les autorités locales viennent chasser les vendeurs ambulants. Habiba peut alors se cacher dans le local de sa nouvelle amie, la vendeuse de charbon.

Malgré l'exclusivité dont bénéficie Habiba à Sidi Maarouf, le pouvoir d'achat des habitants n'y est pas très élevé et la clientèle n'est pas suffisamment diversifiée. Elle décide donc d'y aller seulement les jours où la clientèle est abondante: le vendredi et le samedi. Les dimanches, c'est au tour de son emplacement précédent sur la route principale qui longe la mosquée Lhamd, non loin du souk de Sidi Messaoud. Pour le reste de la semaine, elle se rabat 
désormais sur son emplacement près de la gare ferroviaire de Nassim, dans la zone industrielle de Sidi Maarouf. Cet endroit est particulièrement rentable parce qu'il se trouve en plein zone industrielle, non loin de la gare de Nassim et à quelques mètres du quartier résidentiel comportant principalement des logements sociaux. Sa clientèle, composée d'ouvrières, de voyageurs et des habitants avoisinants, y est donc beaucoup plus diversifiée.

Il reste pourtant un inconvénient majeur à s'installer dans cette zone industrielle. Elle est bien plus souvent confrontée aux agents de police qu'au souk de Sidi Maarouf. Il lui a fallu développer un arsenal de stratégies qui lui permettent de neutraliser leurs colères. La méthode la plus efficace explique t-elle, c'est de les amadouer en jouant la victime, en pleurant, en suppliant à haute voix, racontant qu'elle est veuve et qu'elle doit nourrir ses enfants orphelins. Un accord tacite d'un tout autre ordre semble pourtant s'être établi au fil du temps entre Habiba et les agents habitués de l'endroit. Une scène qui se déroule sous nos yeux en est la parfaite illustration. Debout près du stand de Habiba, nous retenons notre souffle lorsqu'un agent de police se dirige vers elle d'un pas languissant. Nullement déstabilisée, Habiba se met à fouiller dans les poches de son tablier et en sort deux cigarettes qu'elle lui tend en échange d'un dirham. Elle attend que l'agent, complètement indifférent à notre présence, se soit éloigné avant de renchérir : «Avec moi, ces policiers ont un comportement différent. Ils me connaissent bien maintenant. Vous savez que d'ici j'observe tout ce qui se passe sur la route... je suis témoin de leurs manigances avec les conducteurs ». Habiba cligne des yeux et en faisant de la main le signe de l'argent, nous précise qu'eux aussi sont mal placés pour juger ses pratiques informelles.

\section{Le bidonville de Bachkou: stratégique par sa marginalité}

Vendredi, il est $11 \mathrm{~h}$ du matin au Bidonville de Bachkou. Assises par terre au côté de Mina, nous observons la misère, les amas de déchets, l'état vétuste des maisons et nous ne pouvons nous empêcher de nous demander pourquoi elle continue à venir, tous les vendredis, à ce même endroit qui, vu de l'extérieur, ne semble certainement pas propice à faire de bonnes affaires. Pourquoi son père avait-il choisit cet endroit? Pourquoi n'ont-ils jamais changé d'emplacement?

C'est d'un regard qui traduit l'incompréhension que Mina répond à cette question. Visiblement ces deux-là ne savent rien de la réalité du métier. N'est-il pas évident que le bidonville de Bachkou est l'emplacement idéal ?

Bachkou offre à Mina et à son père un type de client bien précis. C'est une clientèle chaabiya (populaire) majoritairement d'origine rurale qui, fuyant la campagne, est venue s'installer en ville. Or c'est cette clientèle qui consomme des produits ruraux comme le petit-lait. C'est également une clientèle qui n'a pas peur de consommer des produits achetés dans la rue. En se 
déplaçant en dehors du bidonville, Mina risquerait d'être confrontée à la peur que le consommateur citadin a de ce qui se vend dans la rue et à des habitudes alimentaires différentes. Les citadins ne perpétuent même plus la tradition du couscous le vendredi et se montrent méfiants envers les produits campagnards se plaint-elle. Ainsi, les marchandes qui vont en ville se dirigent avant tout vers les quartiers populaires et les bidonvilles à la rencontre de leur clientèle.

Situé dans un bidonville en plein quartier résidentiel, l'emplacement de Bachkou est également une sorte d'enclave protégée des hamlat ${ }^{40}$. Mina sait que ces campagnes policières visent principalement les vendeurs de légumes et de fruits qui, de leurs grandes charrettes, bloquent la circulation sur la route et salissent la voie publique. Mais elle sait également qu'en cas de hamla, bien qu'une vendeuse de lben ne dérange pas beaucoup et ne produise pas de déchets, elle peut se voir confisquer tout son matériel. Souvent «c'est parce que l'ordre vient d'en haut » et d'autres fois c'est parce que les habitants des quartiers résidentiels se plaignent que les hamlat sont lancées. A Bachkou, un espace emprunt d'une urbanité informelle, l'activité de Mina peut plus facilement se développer.

De plus, Bachkou est relativement facile d'accès. Privilégiée, car propriétaire d'une charrette et d'un mulet que conduit son père et qui lui permet de déplacer tout son matériel de vente qui n'est pas léger, Mina peut s'aventurer bien plus profondément en ville que ses collègues. En même temps, ce moyen de transport rural étant difficilement admis en ville, Mina doit se limiter aux marges de cette dernière, à ses quartiers périphériques, en empruntant autant que possible les pistes en terre battue qui y mènent.

A Bachkou, enfin, elle tire profit de son réseau de connaissances pré-établi pour garder ses produits au frais. Transporter le lben sur de grandes distances n'est pas possible. Le laisser reposer au soleil pendant des heures n'est pas non plus recommandable. La meilleure solution est de garder le produit au frais grâce à une bouteille d'eau glacée plongée dans la jarre. Cette bouteille devant être remplacée régulièrement, Mina dépend de la bonne volonté des habitants de Bachkou prêts à garder quelques bouteilles de rechange dans leur congélateur. Une bonne volonté qui se laisse aisément entretenir par une motte de beurre, une bouteille de lben voire même un peu d'argent. Pour 20 centimes la pièce, les habitants du bidonville la ravitaille également en bouteilles de plastique vide. Peut-on rêver d'un meilleur endroit de vente?

\section{Conclusion}

«Lorsque j’étais jeune, je ne sortais jamais voir la ville (lmdina). Ma sœur puis mon mari ne me laissaient pas quitter le douar. La nuit, je voyais les lumières de la ville. Elle me semblait toute proche. La toute première fois que je me suis rendue en ville, je me rappelle que c'était

\footnotetext{
${ }^{40}$ Désigne les campagnes policières.
} 
pour aller chez le médecin. Le petit était malade. On a pris la route à l'aube pour aller à pied jusqu'à Ain Chock, près de la khayriya (orphelinat). Je me suis alors rendue compte que la ville était beaucoup plus loin que je ne pensais. Nous ne sommes arrivés qu'à $8 \mathrm{~h}$ du matin. La salle d'attente était déjà pleine. »

Depuis, la vie de Habiba a bien changé. Par son petit commerce ambulant, elle contribue à la plus grande partie du budget familial. Casablanca n'est plus cette masse lumineuse qu'elle observait au loin. Ayant pris possession des quartiers les plus périphériques de la ville, c'est avec envie qu'elle pense à tous ces emplacements stratégiques qu'elle pourrait découvrir en s'aventurant plus profondément en ville. Pourtant, de la même manière que Mina et Rahma, Habiba n'en fréquente que la ceinture extérieure. Les trois femmes n'associent plus Bab Marrakech au centre de la ville. Celle-ci s'agrandissant, l'ancien centre s'est éloigné des habitants de Sidi Messaoud. Il s'est déplacé vers la périphérie de la ville : vers Aïn Sbaa, Aïn Chock, Sidi Maarouf, Sahat Sraghna, Derb Ghellaf, Derb Seltane ${ }^{41}$ et Derb Omar ${ }^{42}$ où elles rendent occasionnellement visite à « la famille » et font, de temps en temps, quelques courses. Garage $\mathrm{Allal}^{43}$ qui se situe en plein cœur de Derb Seltane et qui est une adresse de choix pour faire toutes sortes d'emplettes. Elles y trouvent de tout: du matériel pour le travail, des produits introuvables dans les souks et ces houijat ${ }^{44}$ qui sont de petites gâteries de toutes sortes, des habits et jouets pour les enfants. Elles dépassent rarement la limite de ces quartiers qui symbolisent à leurs yeux Imdina (la ville). Clignant des yeux, Mina explique qu'elle a même entendu dire qu'il était prévu, un jour, de faire de Sidi Messaoud le nouveau centre de la ville. De toute façon, ajoute- t-elle, la ville approche tellement vite, il n'y en a plus pour longtemps.

Pourtant, ce n'est pas par manque de moyens de transport qu'elles ne s'aventurent pas plus loin. Il est, aujourd'hui, beaucoup plus simple d'y accéder qu'avant. Rahma se rappelle bien de l'époque où il $\mathrm{n}$ y avait qu'un petit bus qui faisait une fois par jour l'aller-retour entre le site et la ville. Ensuite, est arrivé le premier grand taxi. C'était celui d'un militaire qui a été rapidement suivi de 7 autres taxis qui pendant de longues années ont fait le trajet entre Sidi Messaoud et Derb Ghellaf. Les commerçants du site peuvent citer chacun des huit noms de conducteurs. Tous, à l'exception de Sidi Driss, sont décédés aujourd'hui. Ils ont été remplacés par plusieurs autres taxis qui relient désormais régulièrement Sidi Messaoud à Derb Ghellaf. Une fois arrivé là, il est possible d'accéder à tous les coins de la ville. Il est également possible de se faire conduire, précise Mina, par un voisin ou une connaissance en voiture. Ces déplacements se font alors rarement par la route principale parce que la plupart des habitants du Douar n'ont pas leurs papiers de voiture en règle. Pour contourner les contrôles policiers,

\footnotetext{
${ }^{41}$ Quartier commercial situé au centre de Casablanca.

${ }^{42}$ Quartier commercial connu par ses vendeurs de tissus et son marché de gros où viennent s'approvisionner les vendeurs.

${ }^{43}$ Ce marché se caractérise par ses prix très bas et ses produits de contrebande.

${ }^{44}$ Littéralement : « petites choses ».
} 
ils préfèrent, en règle générale, prendre la piste qui relie Sidi Messaoud à Sbata. Elle traverse les terrains vagues, permet de se cacher plus facilement et est beaucoup moins contrôlée qu'une route balisée.

Paradoxalement, cette ville qui approche et les moyens de transport qui se multiplient ne sont pas des facteurs qui semblent réellement attirer nos trois vendeuses. Les intrusions en ville se font par ses marges et de façon très ponctuelle. En fin de journée, elles se retrouvent toutes à Sidi Messaoud. Le lieu est alors devenu beaucoup plus calme. Rahma, préférant éviter la tombée de la nuit, a déjà descendu la grille et est rentrée chez elle. Mina, assise sur une chaise devant son local, observe les allées et venues qui se font plus rares et guette le retour des clients du matin qui, en rentrant chez eux, s'arrêtent souvent pour emporter du lait et du beurre pour le reste de la famille. Comme tous les soirs, Habiba confie sa petite charrette à bras au vieux gardien du marabout qui la gardera jusqu'au lendemain pour dix dirhams par semaine. Le bruit des ferronniers ne se faisant plus entendre, une certaine sérénité s'impose. Sidi Messaoud redevient le lieu de culte et le mausolée, du haut de sa colline, reprend son allure singulière empreinte du mystère que lui confère le silence mystique qui l'entoure soudainement.

\section{Ouvrages cités}

AZAR Mélina, «Deux exemples d'opérations de logement social au Maroc : al Anbar-Massira II à Marrakech et Nassim, Ville Nouvelle à Casablanca ", dans Archibat : revue maghrébine d'aménagement de l'espace et de la construction . - N. 3 (2001) . - p. 78-81

BENSABER Bouchra, «Les saints de Casablanca : Sidi Messaoud, Sidi Mohamed Moul Essabiane, Sidi Mohamed Mers Soltane et tous les autres noms de Casablanca », La Gazette du Maroc, 25 Septembre 2006

BENYAKHLEF Brahim; BOUKHEMIS Kaddour; ZEGHICH Anissa, 2005, «Croissance périurbaines et émergence de nouvelles formes d'urbanisation en zones périurbaines de la ville d'Annaba », dans Dynamiques des territoires et des sociétés, les documents de la MRSH de Caen, $\mathrm{N}^{\circ} 15$, P.49-66

ETIENNE Bruno, « «Magie et thérapie à Casablanca», dans: Sourai Christine (dir.), Le Maghreb musulman en 1979. Paris : 1981. 412p. p.263-284

MONNET Jérôme, «Ambulantage : représentations du commerce ambulant ou informel et $\begin{array}{lllll}\text { métropolisation », } & \text { Cybergeo, } & \text { Nr.355, } & 17 & \text { Octobre }\end{array}$ http://www.cybergeo.eu/index2683.html 